\title{
Références bibliographiques du dossier « musique et éducation »
}

Hélène Beaucher

\section{OpenEdition}

1 Journals

Édition électronique

URL : https://journals.openedition.org/ries/5991

DOI : 10.4000/ries.5991

ISSN : 2261-4265

Éditeur

France Education international

\section{Édition imprimée}

Date de publication : 1 septembre 2017

ISBN : 978-2-85420-615-9

ISSN : $1254-4590$

\section{Référence électronique}

Hélène Beaucher, «Références bibliographiques du dossier « musique et éducation » », Revue internationale d'éducation de Sèvres [En ligne], 75 I septembre 2017, mis en ligne le 01 septembre 2017, consulté le 25 juin 2021. URL : http://journals.openedition.org/ries/5991 ; DOI : https://doi.org/ $10.4000 /$ ries.5991 


\title{
Références bibliographiques du dossier « musique et éducation "
}

\author{
Hélène Beaucher \\ Centre de ressources et d'ingénierie \\ documentaires, CIEP
}

\begin{abstract}
L'étude scientifique du cerveau et l'intérêt pour les activités artistiques en neurosciences sont très récents. Ils montrent que la musique s'enracine profondément dans notre cerveau et suggère de nombreuses implications pour l'éducation. La pratique musicale a-t-elle des effets positifs sur les compétences non artistiques? Quelle est la place de la musique dans les politiques publiques? Quelles sont les expériences en faveur de l'accès des jeunes à la musique ?

Cette bibliographie concerne en majorité des travaux produits ces dix dernières années sur ce sujet. La première partie vise à apporter des éléments de réflexion sur les enjeux sociaux, culturels et politiques de la musique. La deuxième partie propose des ouvrages et articles sur le thème "cerveau, musique et émotions ». La troisième partie présente une sélection d'études examinant les impacts de la musique sur les compétences cognitives, comportementales et sociales. Les dernières parties fournissent un aperçu des politiques, pratiques et dispositifs d'apprentissage de la musique, avec un focus sur le développement international des orchestres et chorales d'enfants. Les résumés sont, pour l'essentiel, ceux des éditeurs ou des revues. Bibliographie arrêtée le 15 juillet 2017.
\end{abstract}

\section{ENJEUX SOCIAUX, CULTURELS ET POLITIQUES DE LA MUSIQUE}

\section{BARENBOÏM Daniel, La musique éveille le temps, Fayard/Paris, 2008, 216 p.}

La musique exige un parfait équilibre entre intellect, émotion et tempérament. Daniel Barenboïm analyse la façon dont la perception de la musique s'inscrit à la fois dans le corps, dans l'esprit et dans la vie sociale. Il a fondé et dirige le West-Eastern Divan Orchestra, un orchestre qui réunit de jeunes instrumentistes d'Israël, des États arabes voisins et des Territoires palestiniens pour promouvoir le dialogue et la paix.

BONINI BARALDI Filippo, Tsiganes, musique et empathie, Maison des sciences de l'homme/Paris, 2013, 360 p. + DVD-ROM

Les recherches de l'auteur chez les Tsiganes de Transylvanie (Roumanie) explorent le lien entre musique, émotion et empathie, et se concentrent sur différents contextes sociaux, où musique et pleurs vont de pair. L'approche de ces thèmes est interdisciplinaire (ethnomusicologie, anthropologie et psychologie cognitive). L'ouvrage invite à penser l'émotion musicale comme une forme d'identification, de projection, d'empathie avec des êtres, des «agents » que la musique véhicule et porte en elle.

\section{DELORENZO Lisa C., Giving voice to democracy in music education: diversity} and social justice in the classroom, Routledge/New York, 2015, 286 p.

L'ouvrage traite de l'éducation musicale comme moyen de façonner la conscience démocratique, et voit dans la démocratie une dimension vitale de l'enseignement de la musique. Les différentes 
contributions adoptent des perspectives politiques, pédagogiques et humanistes, pour aborder l'enseignement de la musique en tant que pratique démocratique à la fois à l'école et dans la formation des enseignants.

\title{
ELLIOTT David J., SILVERMANN Marissa, Music matters: a philosophy of music
} education, Oxford university press/Oxford, 2014, 568 p.

Pourquoi la musique est-elle si importante dans nos vies? Comment a-t-elle le pouvoir de nous transformer? Fondé sur un large éventail de sources, l'ouvrage propose une véritable philosophie de l'éducation à et par la musique, intégrant les aspects socioculturels, artistiques, participatifs et éthiques des valeurs de la musique, de l'éducation, de la pratique et de l'écoute musicale, de sa compréhension, des émotions qu'elle suscite, ainsi que de la musique en communauté.

GAULIER Armelle, GARY-TOUNKARA Daouda, DJEBBARI Élina, et al., Musique et pouvoir, pouvoirs des musiques dans les Afriques, Afrique contemporaine, $n^{\circ} 254,2015$, p. 7-126

Ce dossier explore les relations entre musique, migration et politique sur le continent africain et dans les diasporas. Des chansons de l'exil aux chants de la migration, des circulations artistiques transcontinentales aux médiateurs d'échanges culturels, en passant par la production de discours alternatifs d'émancipation, les liens entre musique, migration et politique sont décryptés.

POMPIDOR Henri, "Pour une sociologie du chant choral : contribution à l'analyse sociale et culturelle des pratiques chorales ", L'éducation musicale, octobre 2016, n. p. [en ligne]

150 Vecteur majeur de socialisation, le chant choral représente l'un des moyens de transmettre les normes et les valeurs constitutives de la société et des groupes sociaux qui la composent. Il a été et reste utilisé pour unifier les individus autour d'une même langue, d'une même culture ou d'une même nation. L'article propose d'identifier un nouveau champ de recherches, dont l'objet est d'étudier les modes et les significations des pratiques chorales dans les sociétés. [http://bit.ly/2rSNhlB]

\section{TURINO Thomas, Music as social life: the politics of participation, University of Chicago Press/Chicago, 2008, 280 p., + CD}

Dans le monde entier et à travers l'histoire, on a utilisé la musique pour exprimer émotions intérieures, rejoindre le divin ou endormir les bébés... Initiation au pouvoir de la musique, l'ouvrage analyse les raisons pour lesquelles la musique et la danse sont souvent au cour de nos expériences personnelles et sociales les plus profondes. L'auteur distingue le cas des performances censées être "présentationnelles ", opposé aux performances destinées à être "participatives ", incitant à réévaluer la notion de genre musical à partir de la fonction sociale et des contenus de la performance.

\section{La musique dans les sociétés traditionnelles}

\author{
GUILLEBAUD Christine, Le chant des serpents musiciens itinérants du Kerala, \\ CNRS/Paris, 2008, 384 p. + DVD-ROM \\ Officiants de rituels domestiques, chanteurs au porte-à-porte, contractuels à la radio d'État, \\ etc., les musiciens itinérants du Kerala (Inde du Sud) pratiquent leur art selon des codes sans \\ cesse redéfinis. Pour comprendre la vie musicale de ces musiciens, il est indispensable de consi- \\ dérer leur musique «dans tous ses états» (pratiques, discours, espaces et relations sociales).
}


L'auteure propose de reconsidérer les rapports entre musique et société, en prenant comme axe d'observation la mobilité des musiciens et leur relation commune, via ce service musical et rituel de traitement des maux et des infortunes, à des commanditaires de caste supérieure.

\section{LEGRAIN Laurent, Chanter, s'attacher et transmettre chez les Darhad de} Mongolie, Paris, Centre d'études mongoles et sibériennes - École pratique des hautes études/Paris, 2015, 394 p. + CD audio

Aux confins septentrionaux du pays où vivent les Darhad, l'attachement au chant est perçu comme une dimension pérenne de l'ethos culturel. Inscrivant ses analyses dans la temporalité de l'histoire socialiste, l'auteur trace les contours du monde sonore mongol. Il examine les différents dispositifs par lesquels les enfants sont encouragés à se positionner dans le continuum sonore, parmi lesquels les cours de chant dispensés à l'école et le gardiennage du troupeau.

LONCKE Sandrine, Geerewol: musique, danse et lien social chez les Peuls nomades woddabe du Niger, Société d'ethnologie/Paris, 2015, 416 p. + DVD-ROM

Au cœur du Sahel nigérien, des milliers de Peuls nomades wodaabe se réunissent chaque année pour un rassemblement cérémoniel, dont le rituel est appelé geerewol. Deux fractions de lignages différents se livrent une guerre dont les seules armes sont le chant et la danse. Comment la musique et la danse sont-elles l'expression esthétique de différentes manières d'être ensemble? Pourquoi le rituel et la performance artistique collective sont-ils des espaces privilégiés pour faire société ? L'enquête conduit au cœur des représentations culturelles et des conceptions esthétiques de cette société ouest-africaine d'éleveurs nomades.

\section{MUSIQUE, CERVEAU, ÉMOTIONS : L'APPORT DES NEUROSCIENCES ET DES SCIENCES COGNITIVES}

BIGAND Emmanuel dir., TILMANN Barbara dir., PERETZ Isabelle dir., et al., "The neurosciences and music V: cognitive stimulation and rehabilitation", Annals of the New York Academy of sciences, $n^{\circ}$ 1337, mars 2015, p. 1-271

Ce volume propose un panorama complet des études neuroscientifiques actuelles concernant les effets de la musique sur le cerveau, et des implications sociales qui en découlent pour l'éducation et la santé.

\section{BIGAND Emmanuel dir., Le cerveau mélomane, Belin/Paris, 2013, 220 p.}

Notre cerveau est mélomane dès la naissance. L'auteur se penche sur l'universalité des émotions induites par la musique et présente les plus récentes découvertes scientifiques sur les interactions entre langage et musique, sur l'influence bénéfique de cette dernière sur les capacités cognitives, et sur la plasticité cérébrale. Les pratiques musicales touchent au plus profond de notre cerveau, en coordonnant l'activité de nombreux circuits corticaux et sous-corticaux qui sont associés à des expériences cognitives et affectives ayant de très fortes implications pour la mémoire.

BIGAND Emmanuel dir., HABIB Michel dir., BRUN Vincent dir., Musique et cerveau: nouveaux concepts, nouvelles applications, Sauramps Médical/ Montpellier, 2012, 135 p.

La littérature scientifique s'est enrichie ces dernières décennies de très nombreux travaux précisant les effets de la musique sur le cerveau. L'objectif de cet ouvrage est d'illustrer ces connaissances et ces débats pour quelques-uns des domaines les plus marquants de la recherche scientifique médicale, avec des applications thérapeutiques maintenant reconnues, mais aussi avec un large champ de perspectives pour l'avenir. 
HABIB Michel, BESSON Mireille, "Langage, musique et plasticité cérébrale: perspectives pour la rééducation ", Revue de neuropsychologie, 2008, vol. 18, $n^{\circ} 1-2, p .103-126$

L'expertise musicale est associée à des particularités qui ont pu être révélées grâce aux moyens de neuro-imagerie. Elles concernent plusieurs régions toutes impliquées soit dans le contrôle moteur du geste soit dans les fonctions audio-perceptives. L'apprentissage de la musique possède des effets plus généraux sur la plasticité cérébrale. Les implications sont discutées, avec comme perspective l'utilisation de l'entrainement musical chez des enfants souffrant de troubles spécifiques d'apprentissage du langage et de la lecture.

KOLINSKY Régine dir., MORAIS José dir., PERETZ Isabelle dir., Musique, langage, émotion : approche neuro-cognitive, Presses universitaires de Rennes/Rennes, mai 2010, 127 p.

À la croisée de plusieurs disciplines - psychologie, psychologie cognitive, psycholinguistique, neuropsychologie, neurosciences, musique - cet ouvrage illustre le foisonnement récent des connaissances neuroscientifiques sur la musique et ses relations avec le langage et les émotions. Les auteurs abordent la perception des structures musicales dans ses relations avec le langage, le rôle et l'importance des émotions dans la musique ainsi que les éventuels dysfonctionnements du traitement de ces émotions.

KRAUS Nina, SLATER Jessica, THOMPSON Elaine C., et al., « Music enrichment programs improve the neural encoding of speech in at-risk children ", The journal of neurosciences, 2014, vol. 4, $n^{\circ}$ 36, p. 11913-11918 [en ligne]

152 L'équipe de chercheurs de l'école de communication de la Northwestern University de Chicago a analysé in situ l'évolution de la perception sonore par le cerveau des enfants participant pendant au moins deux ans à un programme de formation musicale. L'étude fournit les preuves concrètes de l'évolution neurobiologique du cerveau de ces enfants. Elle démontre que l'apprentissage de la musique peut littéralement remodeler le cerveau d'un enfant de façon à améliorer sa réception sonore, ce qui améliore automatiquement ses aptitudes d'apprentissage et d'acquisition du langage. [http://bit.ly/1w919qx]

\section{LECHEVALIER Bernard dir., PLATEL Hervé dir, Le cerveau musicien : neuro-} psychologie et psychologie cognitive de la perception musicale, De Boeck Supérieur/Bruxelles, 2010, 332 p.

Comment percevons-nous la musique ? L'ouvrage fait explicitement le lien entre les études de psychologie cognitive et de neurosciences cliniques et fondamentales sur la perception musicale. Il permet de comprendre comment la musique est un stimulant cognitif et cérébral et une forme d'expression particulière de l'intelligence, qui trouvent leur source dans le besoin de notre cerveau d'associer expériences sensorielles, motrices et émotionnelles.

\section{LEMAN Marc, Embodied music cognition and mediation technology, MIT Press/ Cambridge MA, 2008, 297 p.}

S'appuyant sur des travaux issus de la recherche en informatique, psychologie, neurosciences et musicologie, l'ouvrage propose une théorie sur l'incarnation de la cognition musicale. Le corps humain agirait comme un médiateur entre l'esprit et le monde physique, musical. L'expérience musicale serait basée sur une interaction entre le la musique et le corps. Il présente un modèle qui décrit la relation entre un sujet humain et son environnement; il analyse le couplage perception/action, explore différents niveaux d'engagement du corps et examine les applications possibles. 
TRAINOR Laurel, HANNON Erin, " Musical development ", in The psychology of music, DEUTSCH Diana dir., Elsevier/London, 2013, p. 423-497

Les auteurs passent en revue les phases essentielles du développement des aptitudes musicales en détaillant les interactions musicales précoces des nourrissons et de leurs parents, puis en montrant comment se forgent, au fil des premières années, les fondements des aptitudes mélodiques, rythmiques et harmoniques. L'impact de la formation musicale sur les aptitudes psychologiques générales est également abordé.

TREHUB Sandra, TRAINOR Laurel, «Les stratégies d'écoute chez le bébé : origine du développement de la musique et de la parole ", in Penser les sons: psychologie cognitive de l'audition, BIGAND Emmanuel dir., MCADAMS Stephen dir., PUF/Paris, 1994, p. 299-347

Les auteurs analysent en détail toutes les aptitudes musicales précoces des nourrissons dans les domaines de la perception du rythme, de la hauteur et de l'harmonie. On y découvre que les nourrissons sont de véritables musicologues, bien avant l'heure.

\title{
LES IMPACTS DE L’ÉDUCATION ET DE LA PRATIQUE MUSICALES
}

\begin{abstract}
CATTERALL James C., DUMAIS Susan A., HAMPDEN-THOMPSON Gillian, The arts and achievement in at-risk youth: findings from four longitudinal studies, National endowment for the arts/Washington, 2012, 27 p. [en ligne]

La pratique d'activités artistiques améliore-t-elle les résultats scolaires et les compétences comportementales et sociales des enfants et des jeunes défavorisés? Les auteurs examinent les données issues de quatre grandes études longitudinales nord-américaines pour comparer les résultats obtenus par les élèves ayant une pratique assidue des arts (musique, danse, théâtre, arts visuels) à l'école ou hors de l'école avec ceux des élèves ne pratiquant pas ou peu une activité artistique. Les résultats suggèrent que l'éducation artistique peut constituer une voie importante pour améliorer les résultats des jeunes défavorisés. [http://bit.ly/10Cm908]
\end{abstract}

HALLAM SUsan, The power of music: a research of the impact on the intellectual, social and personal development of children and young people, International Music education research centre - iMERC/London, 2015, 168 p. [en ligne] Ce rapport fournit un panorama des bénéfices de la musique sur le développement intellectuel, social et personnel des enfants et des jeunes. Il explore les preuves fournies par les neurosciences et la littérature de recherche de l'impact de l'apprentissage de la musique sur le développement du langage, le développement intellectuel, la créativité, la confiance en soi, la sensibilité émotionnelle, la concentration, le travail en équipe, et le bien-être. Une pratique musicale assidue contribue à améliorer toute une gamme de compétences non musicales.

[http://bitlly/2sayyWV]

\section{LAURET Jean-Marc, L'art fait-il grandir l'enfant ? Essai sur l'évaluation artis- tique et culturelle, Éditions de l'Attribut/Toulouse, 2014, 160 p.}

Souvent reléguée après les apprentissages fondamentaux, l'éducation artistique et culturelle ne va pas de soi. Ses objectifs peuvent diverger : réussite scolaire, intégration professionnelle ou épanouissement personnel. L'ouvrage propose une synthèse des recherches menées depuis une trentaine d'années, principalement aux États-Unis, en matière d'évaluation artistique et culturelle. Il privilégie une approche qualitative, en s'appuyant sur les compétences forgées par l'éducation artistique : créativité, imagination, confiance personnelle, concentration, faculté d'apprentissage, estime de soi, ouverture à l'autre, prise de conscience de son environnement. 
WINNER Ellen, GOLDSTEIN Thalia R., VINCENT-LANCRIN Stéphane, L'art pour l'art? L'impact de l'éducation artistique, OCDE/Paris, 2014, 300 p.

L'éducation artistique est souvent considérée comme un moyen de développer des compétences essentielles pour l'innovation : pensée critique et créative, motivation, confiance en soi, capacité à communiquer et coopérer, mais aussi des compétences dans des disciplines scolaires non artistiques. L'ouvrage a pour objectif d'évaluer l'impact de l'éducation artistique sur un vaste éventail de compétences, en examinant de manière critique l'ensemble des travaux de recherche menés actuellement dans le domaine de l'éducation et de la psychologie. Les différents types d'éducation artistique étudiés comprennent l'enseignement des arts dans le cadre scolaire (musique, arts plastiques, théâtre et danse), et hors du cadre scolaire.

\section{Impacts sur les facultés cognitives et comportementales}

\section{LECOQ Aurélie, SUCHAUT Bruno, L'influence de la musique sur les capacités} et les apprentissages des élèves en maternelle et en cours préparatoire, IREDU/ Dijon, 2012, 6 p.

Cette note expose les résultats d'une recherche dans les classes de grande section maternelle, dont l'objectif était de mesurer les effets de la pratique musicale sur le développement cognitif et les acquisitions scolaires. L'expérimentation confirme l'efficacité des activités musicales sur les acquisitions scolaires des jeunes élèves même si les mécanismes de transfert entre musique, capacités cognitives et performances scolaires n'ont pu être clairement mis à jour. [http://bit.ly/1PFX003]

SCHELLENBERG E. Glenn, "Music lessons enhance IQ ", Psychological Science, vol. 15, $n^{\circ}$ 8, 2004, p. 511-514 [en ligne]

Cette étude expérimentale menée auprès d'enfants de six ans avait pour but de tester l'hypothèse selon laquelle la musique rend plus intelligent. 144 enfants de 6 ans ont été répartis de manière aléatoire dans quatre groupes : cours de clavier, cours de chant, cours de théâtre et aucun cours. Le QI des enfants a été testé avant le début des cours et après 36 semaines de cours. L'auteur montre que les cours de musique, par opposition aux cours de théâtre ou à l'absence d'enseignement artistique, induisaient une augmentation des scores obtenus aux tests de QI par les jeunes enfants. [http://bit.ly/1klMv07]

\section{Éducation musicale, lecture et conscience phonologique}

BOLDUC Jonathan, RONDEAU Julie, "Rythmons les apprentissages ! ", Langage et pratiques, 2015, vol. 56, p. 15-22 [en ligne]

De nombreuses études ont documenté l'impact de l'éducation musicale sur le développement des habiletés d'éveil à l'écrit. Des recherches ont notamment établi des corrélations positives et des liens causals significatifs entre le traitement rythmique et les capacités de conscience phonologique au préscolaire. À cette période où les apprentissages ludiques prédominent, la réalisation de comptines combinant rythmes et mots serait un moyen efficace de sensibiliser l'enfant aux unités sonores du langage. [http://bit.ly/2sZuR6u] 
FLAUGNACCO Elena, LOPEZ Luisa, TERRIBILI Chiara, et al. " Music training increases phonological awareness and reading skills in developmental dyslexia: a randomized control trial », PLoS ONE, 2015, 17 p. [en ligne]

La dyslexie, qui se manifeste par des difficultés de lecture, serait due à un problème d'encodage des sons. La musique favoriserait la synchronisation neuronale pour traiter les tonalités et les rythmes, avec des répercussions positives sur l'audition et le langage. Les chercheurs ont testé le bénéfice d'une cure de musique chez des enfants dyslexiques. Des séances hebdomadaires de musique rythmée pendant quelques mois améliorent considérablement les capacités de lecture des enfants dyslexiques. Il y a un transfert de compétence au sein du cerveau, du rythme vers l'habilité à discerner les sons et donc à lire correctement. [http://bit.ly/2sup|Sa]

\section{Compétences sociales}

KIRSCHNER Sebastian, TOMASELLO Michael, "Joint music making promotes prosocial behavior in 4-year-old children ", Evolution and human behavior, vol. 31, 2010, p. 354-364 [en ligne]

Dans les cultures traditionnelles, la musique et la danse font souvent partie intégrante de cérémonies de groupe importantes; l'une des hypothèses est que la musique favoriserait le lien social, la cohésion du groupe et la coopération. Des enfants de 4 ans participant à une formation musicale en groupe pendant dix mois ont été comparés un groupe témoin de même statut socio-économique. Les enfants du groupe de musique étaient plus susceptibles d'aider spontanément un autre enfant. Les résultats suggèrent que la formation musicale en groupe facilite le développement des compétences prosociales. [http://bit.ly/2uhz578]

PEARCE Eiluned, LAUNAY Jacques, DUNBAR Robin I. M., "The ice-breaker effect: singing mediates fast social bonding ", Royal society open science, octobre 2015, $n^{\circ}$ 2, 9 p. [en ligne]

Pendant sept mois, des groupes d'adultes suivant une fois par semaine les cours de chant choral ont été observés. Dans cette étude, les chercheurs prouvent que cette activité agit à plusieurs niveaux liés à la socialisation : elle exige la coordination, donc l'interaction, et augmente le comportement pro-social et le sentiment d'affiliation. Ils ont mesuré un regain de sentiments positifs et une plus grande tolérance à la douleur, par la production augmentée de deux hormones impliquées dans l'attachement et le sentiment de bien-être : l'endorphine et l'ocytocine. [http://bit.ly/2suoRld]

\section{POLITIQUes ET INITIATIVES D'APPRENTISSAGE DE LA MUSIQUE}

\section{Politique internationale et européenne}

DUDT Simone, "From Seoul to Bonn: a journey through international and European music education policies ", in Listen out: International perspectives on music education, HARRISON Chris dir., HENNESSY Sarah dir., Musik Mark/ London, 2012, p. 126-137 [en ligne]

L'auteure examine les contextes de politique culturelle internationaux et européens pour l'éducation musicale. Elle explore les stratégies développées par l'Unesco en matière d'éducation artistique et fournit un aperçu des politiques en place au niveau de l'Union européenne.

[http://bitlly/2selXgP] 
EMC : European Music Council, La Déclaration de Bonn : une adaptation de l'Agenda de Séoul dans le contexte européen de la musique, EMC/Bonn, 2011, 5 p. [en ligne]

En mai 2011, le Conseil européen de la musique (CEM) a invité des structures actives dans le domaine de l'éducation musicale à discuter de la mise en ouvre des objectifs pour le développement de l'éducation artistique inclus dans l'Agenda de Séoul. La Déclaration de Bonn reflète les arguments de l'Agenda et interprète ces objectifs en mettant l'accent sur l'enseignement de la musique en Europe. [http://bit.ly/2siYdfg]

\section{UNESCO, L'Agenda de Séoul : objectifs pour le développement de l'éducation} artistique, UNESCO/Paris, 2010, 11 p. [en ligne]

Résultant de la seconde Conférence mondiale de l'UNESCO sur l'éducation artistique, l'Agenda de Séoul appelle l'attention des États membres de l'Unesco, de la société civile, des organisations et communautés professionnelles, sur la nécessité de reconnaître les objectifs, d'appliquer les stratégies proposées et de mettre en œuvre les actions, dans un effort concerté pour parvenir à une éducation artistique de grande qualité qui profite aux enfants, aux jeunes et aux apprenants de tous âges. [http://bit.ly/2rmvl61]

\section{Études transnationales}

COX Gordon dir., ROBIN Stevens dir., The origins and foundations of music education cross-cultural historical studies of music in compulsory schooling, Continuum/London, 2010, 256 p.

156 L'ouvrage explore les origines et les fondements de l'éducation musicale et examine son intégration dans le curriculum de l'enseignement scolaire en Europe (Grande-Bretagne, France, Allemagne, Irlande, Norvège, Espagne), en Amérique du Nord (Canada, États-Unis), en Amérique latine (Argentine, Cuba), en Afrique, et en Asie-Pacifique (Australie, Chine, Japon, Afrique du Sud). Les différentes contributions analysent, dans le contexte politique et social de chaque pays, les objectifs et contenus du programme d'enseignement, les méthodes pédagogiques, la formation des enseignants et les expériences des élèves.

Eurydice : réseau d'information sur l'éducation en Europe, L'éducation artistique et culturelle à l'école en Europe, EACEA: Education audiovisual and culture executive agency/Bruxelles, septembre 2009, 106 p. [en ligne]

L'étude examine la place de l'éducation artistique et culturelle dans les programmes scolaires de trente pays européens; elle en présente les objectifs, l'organisation, l'offre d'activités extrascolaires ainsi que les initiatives en faveur du développement de l'éducation artistique et culturelle. [http://bit.ly/2u16iDE]

DURAN Lucy éd., Growing into music: a multicultural study of musical enculturation in oral traditions, School of oriental and african studies, London University/London, 2012, DVD, 1:05 mm [en ligne]

Réalisé dans le cadre du projet Growing Into Music, ce film explore la façon dont les enfants acquièrent les répertoires et les techniques de la musique dans différentes cultures de tradition orale (Inde, Mali, Guinée, Azerbaïdjan, Cuba, Venezuela). L’apprentissage se déroule parfois au sein des familles, et implique différentes pédagogies, allant des leçons individuelles à l'apprentissage en groupe, par jeu, osmose, imitation ou encore à l'école. Les enfants apprennent le langage corporel de la musique avant même d'apprendre la technique musicale. [http://bitly/2tTN6Km] 


\title{
Politiques \\ et pratiques nationales
}

\begin{abstract}
BMBF: Bundesministerium für Bildung und Forschung, Instrumentalunterricht in der Grundschule: Prozess- und Wirkungsanalysen zum Programm Jedem Kind ein Instrument, BMBF/Berlin, 2015, 292 p. [en ligne]
\end{abstract}

Dispensé dans des écoles primaires, en Rhénanie du Nord - Westphalie depuis 2008, puis à Hambourg, le programme JeKi - Jedem Kind ein Instrument a pour objectif de permettre à chaque enfant d'apprendre à jouer d'un instrument de musique. Il repose sur un partenariat local entre un établissement scolaire et un conservatoire de musique, dans une logique d'initiation à la pratique instrumentale, vocale ou chorégraphique, en groupe. Ce rapport présente les résultats scientifiques des projets de recherche sur JeKi financés par le BMBF selon quatre perspectives : les effets de l'enseignement instrumental à l'école primaire, la coopération entre l'école de musique et les enseignants, la qualité de l'enseignement. [http://bit.ly/2svfpj6]

OFSTED: Office for standards in education children's services and skills, Music in schools: what hubs must do, OFSTED/London, 2013, 23 p. [en ligne]

Les Music Education Hubs ont été mis en place en 2012 dans le cadre du plan national d'éducation musicale britannique. Ces centres agissent localement, en regroupant et coordonnant les partenariats entre écoles, autorités locales, structures associatives et professionnelles, afin de répondre aux besoins d'un territoire ou d'une communauté. Le rapport précise que les Music Hubs ont insufflé une vitalité et une énergie nouvelles ainsi qu'une méthode collaborative dans le travail avec les jeunes. Il souligne de grandes disparités entre les différentes régions. [http://bitlly/2uyyrSO]

UIBEL Stefanie, "Education through music - the model of the Musikkindergarten Berlin ", Annals of the New York Academy of Sciences, vol. 1252, 2012, p. 51-55

L'auteure présente le Musikkindergarten de Berlin, créé en 2005 à l'initiative du pianiste et chef d'orchestre Daniel Barenboïm. Plus qu'un simple éveil musical, ce jardin d'enfants propose une véritable éducation par la musique. Les enseignants spécialement formés travaillent en étroite collaboration avec les musiciens de la Staatskapelle. Tous les projets développés passent par la musique.

ZHE Ji, "Éduquer par la musique : de l'“initiation" des enfants à la musique classe à la "culture de soi" confucéenne des étudiants ", Perspectives chinoises, 2008, vol. 104, $n^{\circ} 3$, p. 118-129 [en ligne]

La politique et l'éducation sont indissociables dans les tentatives de promouvoir le confucianisme en Chine contemporaine. L'union alors célébrée du sacré, du pouvoir et du savoir s'inscrit en tension avec une modernité d'abord caractérisée par la différenciation des institutions et des valeurs. Partant de ce constat, l'article étudie le cas particulier d'une société engagée depuis 2000 dans l'initiation des enfants à la musique classique et dans la promotion de la « culture de soi » chez les élèves. [http://bit.ly/2rTVc23]

\section{France}

BONNERY Stéphane coordinateur, "L'enseignement de la musique, entre institution scolaire et conservatoires ", Revue française de pédagogie, $n^{\circ} 185,2013$, p. 5-68 [en ligne]

Ce dossier explore les nouvelles formes curriculaires de l'enseignement de la musique, à l'école et dans les conservatoires: dans les cours d'éducation musicale de l'enseignement secondaire ; 
dans les projets partenariaux destinés aux élèves de l'enseignement secondaire ; dans les départements de "musiques actuelles » des conservatoires qui accueillent des adolescents et des jeunes adultes; et dans les dispositifs partenariaux d'éveil musical, destinés aux tout petits enfants et à leurs parents. [http://bit.ly/2sd8iHK]

\title{
DOUCET Sandrine, Les territoires de l'éducation artistique et culturelle : rapport au premier ministre, 2017, 107 p. [en ligne]
}

Comment généraliser les actions d'éducation artistique et culturelle ? Selon l'auteure, l'élément clé de cette démarche est le parcours d'éducation artistique et culturelle (PEAC). Le rapport préconise de construire le parcours en commun en facilitant sa co-construction au plus près du territoire de vie des enfants, sur les temps scolaires, périscolaire et extrascolaires, et de garantir le parcours pour tous, en mettant en valeur l'importance de la gouvernance stratégique à l'échelle régionale. [http://bit.ly/2rqV5d1]

LÉON Jean-Charles coordinateur, "Arts et culture : quels parcours?", Cahiers pédagogiques, $n^{\circ}$ 535, janvier 2017, p. 10-56

Pour donner aux jeunes un égal accès à l'art et à la culture, les derniers textes officiels concernant l'éducation artistique et culturelle mettent l'accent sur la notion de " parcours ", qui doit permettre à l'élève de se constituer une culture personnelle, de développer son habileté artistique et de rencontrer des artistes, des œuvres, des lieux. Une charte pour l'éducation artistique et culturelle a été présentée en juillet 2016. Parmi ses dix principes clés figurent l'exigence de l'accessibilité de l'art à tous, et l'affirmation que la culture est un lien qui permet au jeune de s'inscrire comme sujet du monde.

\section{LES ORCHESTRES ET CHGURS D'ENFANTS ET DE JEUNES}

\section{Le programme El Sistema}

\begin{abstract}
ALEMÁN Xiomara, DURYEA Suzanne, GUERRA Nancy G., et al., "The effects of musical training on child development: a randomized trial of El Sistema in Venezuela », Prevention Science, 2016, p. 1-14

Le curriculum du programme vénézuélien El Sistema met l'accent sur les interactions sociales par l'apprentissage collectif de la musique. L'article décrit les résultats d'une étude visant à évaluer les effets du programme sur le développement des enfants défavorisés fortement exposés à la violence. L'expérimentation conduite dans 16 nucleos (centres de musique) a porté sur un échantillon de 2914 enfants. Les estimations indiquent une amélioration de la maîtrise de soi ainsi qu'une réduction des problèmes de comportement et suggèrent que la participation à El Sistema pourrait jouer un rôle important dans la prévention de la violence et l'acquisition de compétences prosociales. [http://bit.ly/2uhz578]
\end{abstract}

\section{BAKER Geoffrey, El Sistema: orchestring Venezuela's youth, Oxford university press/Oxford, 2014, 362 p.}

L'ouvrage constitue l'une des premières analyses critiques d'El Sistema, souvent présenté comme un moyen de changer le monde. Il questionne les aspects pédagogiques du programme, explorant les problématiques de l'orchestre symphonique. Il interroge la réalité du slogan du système, " action sociale à travers la musique ». Enfin, il analyse la manière dont le programme a réussi ses objectifs, et les rapports entre rhétorique, représentation et réalité. Selon l'auteur, l'idée d'une " action sociale à travers la musique » défendue relève en fait davantage d'une stratégie de marketing. 
CREECH Andrea, GONZALEZ-MORENO Patricia, LORENZINO Lisa, et al., El Sistema and Sistema-inspired programs: a literature review of research, evaluation, and critical debates - second edition 2016, Sistema global/San Diego, 2016, 223 p. [en ligne]

Les dispositifs El Sistema, développés par plus de 90 pays suscitent un nombre croissant de publications. Comment se situent-ils dans le paysage de l'éducation musicale? Les pratiques pédagogiques peuvent-elles être utilisées dans d'autres contextes culturels? Cette revue de la littérature présente l'état des connaissances sur le programme et les dispositifs inspirés d'El Sistema. Elle synthétise les principaux résultats issus des rapports de recherche et d'évaluation. Elle examine le fonctionnement du programme, ses effets sur les compétences, la participation des familles, les aspects pédagogiques, la progression des élèves. Une partie présente les différents débats et critiques que suscite le programme.

\section{Dispositifs d'éducation musicale dans différents pays}

APPEZZATO Ricardo, BRUNO Marta, CAZNOK Yara, et al., "A experiência do Guri Santa Marcelina no ensino musical e inclusão social ", Synergies-Brésil, $n^{\circ}$ 9, 2011, p. 88-97 [en ligne]

À côté du travail d'éducation musicale, Guri Santa Marcelina développe plusieurs actions socio-éducatives, dans le but de transformer les personnes et les communautés. Lancé en 1995 par l'État de São Paulo, ce dispositif brésilien offre depuis sa création à plusieurs milliers d'enfants des cours de solfège et des expériences régulières de pratiques chorales et instrumentale en dehors du temps scolaire. Cet article décrit l'expérience, la mission et les objectifs généraux de ce programme. [http://bit.ly/2sxoN4Z]

CHONG Hyuan Ju, KIM Soo Ji, " Development of school orchestra model in Korean public schools and student's perception of the orchestra experience ", IJEA: International journal of education \& the arts, 2016, vol. 17, $n^{\circ} 35, p .1-17$ [en ligne]

En 2011, le ministère de l'éducation coréen a lancé le projet d'orchestre à l'école SOP (School orchestra project), afin de développer des programmes pour les élèves identifiés à risque dans des régions défavorisées. L'article décrit la mise en place de ce projet mis en ouvre dans 77 établissements d'enseignement primaire et la perception des élèves. Les résultats indiquent que le projet peut offrir aux élèves une opportunité d'acquérir des expériences positives pour la confiance en soi et les relations sociales. [http://bit.ly/2rvB8mw]

COEN Pierre-François, MORA Giorgia, Évaluation des orchestres en classe de Genève : rapport scientifique, Haute école pédagogique de Fribourg/Fribourg, octobre 2015, 63 p. [en ligne]

Les expériences d'Orchestres en classe se développent de plus en plus en Suisse romande. À quoi servent les orchestres de classe? Quels sont les effets et impacts de ce dispositif sur les apprentissages musicaux et les compétences sociales? La confiance et la discipline ? Le but principal de l'orchestre en classe, à savoir le plaisir de faire de la musique ensemble, est plébiscité par tous. [http://bit.ly/2sEzgdY] 
DESLYPER Rémi, ELOY Florence, GUILLON Vincent, et al., Pratiquer la musique dans Démos : un projet éducatif global ?, Observatoire des politiques culturelles/ Grenoble, 2016, 117 p. [en ligne]

Initié en 2010 et coordonné par la Cité de la musique - Philharmonie de Paris, Démos (Dispositif d'éducation musicale et orchestrale à vocation sociale) est un projet de démocratisation culturelle centré sur la pratique musicale en orchestre. De dimension nationale, il est destiné à des enfants habitant des quartiers relevant de la politique de la ville ainsi que des zones rurales insuffisamment dotées en institutions culturelles. Utiliser cette activité comme support d'un accompagnement social plus large, profiter de ce cadre pour renouveler la pédagogie de l'enseignement musical, ou encore construire un modèle d'éducation par l'art destiné à la formation de l'individu et du citoyen constituent autant de facettes du projet Démos, dont ce rapport présente les résultats de l'évaluation globale. [http://bit.ly/2t2aX6X]

GCPH: Glasgow centre for population health, Evaluating Sistema Scotland: initial findings report, GCPH/Glasgow, 2015, 86 p. [en ligne]

Big Noise - Sistema Scotland a pour mission de transformer des vies par la musique d'orchestre. Soutenu par le gouvernement écossais, il est implanté depuis 2008 dans deux quartiers défavorisés de Stirling et de Glasgow. L'accent est mis sur l'apprentissage en groupe et sur une pédagogie musicale essentiellement basée sur le rythme et le corps. Ce rapport présente les résultats de l'évaluation de l'impact de ce programme sur la vie des enfants. Il indique notamment qu'il améliore la confiance, la fierté et l'estime de soi, la santé et le bien-être, ainsi que la fréquentation scolaire, les aspirations des enfants et de leur communauté. [http://bit.ly/2swpECS]

HILLE Adrian, Étude d'impact des orchestres à l'école, Institut Montaigne/Paris, 2010, 39 p. [en ligne]

160 Orchestre à l'école est un projet qui permet à des élèves de collège, notamment en zone d'éducation prioritaire, d'apprendre un instrument pendant trois ans. Cette étude examine l'impact de la participation à un orchestre à l'école sur les résultats académiques, la note de vie scolaire, ainsi que des caractéristiques non-cognitives comme l'attitude envers l'école, l'ambition, l'estime de soi, les rapports sociaux et la confiance envers les autres. L'expérimentation a permis de favoriser de manière significative le développement des capacités cognitives et non-cognitives des élèves. [http://bit.ly/2tFmABq]

\section{LABORDE Denis dir, L'orchestre pour exister ensemble? Démos au cour de la} cité : enquête sur le projet Démos réalisée par l'institut de recherche sur les musiques du monde dans le cadre d'une convention avec la philharmonie de Paris, Philharmonie de Paris/Paris, 2016, 119 p. [en ligne]

Les auteurs interrogent la capacité de modélisation du projet Démos, en analysant la manière dont l'équipe de coordination met en place et anime un système de coopération propre à chaque territoire. En articulant les effets individuels du dispositif avec ses effets globaux, l'étude propose une série d'interrogations pour l'avenir du dispositif. [http://bit.ly/2rdKJNw] 